\title{
Coordination of Ligand-Protected Metal Nanoclusters and Glass Nanopipettes: Conversion of a Liquid-Phase Fluorometric Assay into an Enhanced Nanopore Analysis
}

\author{
Shushu Ding ${ }^{1,2}$, Chunyan Liu ${ }^{1}$, Dingyi Fu², Guoyue Shi ${ }^{1}$, Anwei Zhu*l
}

${ }^{1}$ School of Chemistry and Molecular Engineering, Shanghai Key Laboratory for Urban Ecological Processes and Eco-Restoration, East China Normal University, 500 Dongchuan Road, Shanghai 200241, People's Republic of China

${ }^{2}$ School of Pharmacy, Nantong University, 19 Qixiu Road, Nantong 226001, People's Republic of China

* To whom correspondence should be addressed.

E-mail: awzhu@chem.ecnu.edu.cn

Tel: +86-21-54340042; Fax: +86-21-54340042 
1. Fluorescence emission spectra of Cys and GSH. (Figure S1)

2. Fluorescence emission spectra of BSA-Au NCs upon addition of various amino acids. (Figure S2)

3. TEM image of BSA-AuNCs treated with Cys. (Figure S3)

4. Optical absorption spectra of BSA-AuNCs treated with Cys. (Figure S4)

5. SEM image of polyethyleneimines film. (Figure S5)

6. Optical absorption spectra of the BSA-AuNC film. (Figure S6)

7. Zeta potential of the BSA-Au NC film. (Figure S7)

8. SEM image of the unmodified nanopore tip. (Figure S8)

9. The $I-V$ curves for the modification of glass nanopore. (Figure S9)

10.TEM images of glass nanopore. (Figure S10)

11.Recognition of GSH. (Figure S11)

12.Comparison of the performance of different methods for the determination of cysteine (Table S1) 
1. Fluorescence emission spectra of Cys and GSH.
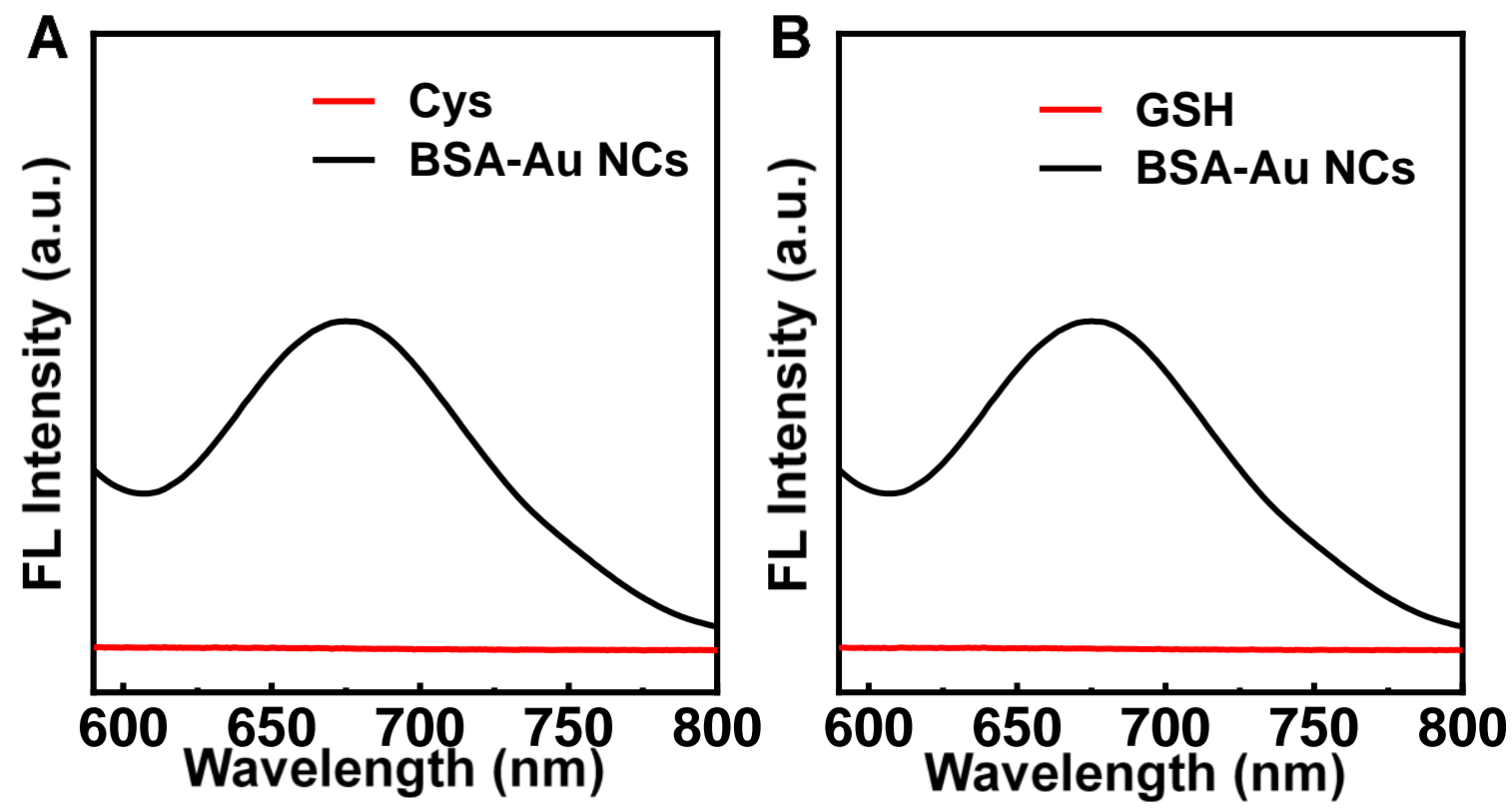

Figure S1. Fluorescence emission spectra of (A) Cys and (B) GSH. $\lambda_{\mathrm{ex}}=405$ nm. 


\section{Fluorescence emission spectra of the BSA-Au NC}

solution

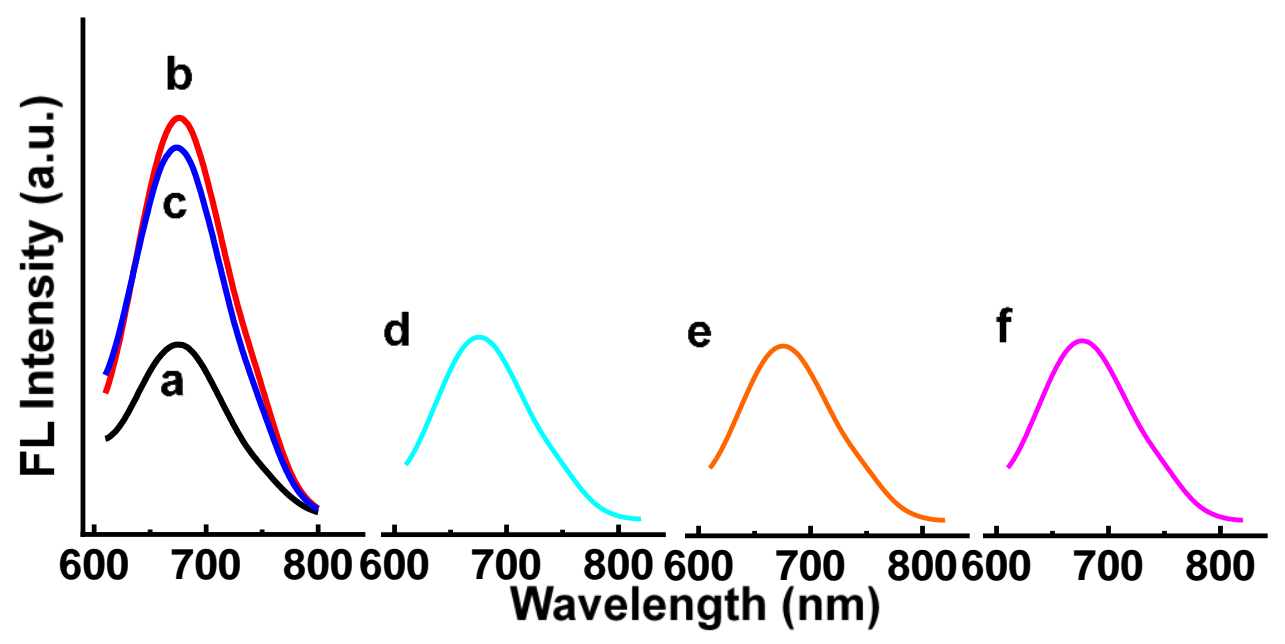

b:<smiles>NC(CS)C(=O)O</smiles>

Cysteine<smiles>CSCCC(NC(C)C)C(=O)O</smiles>

f:<smiles>NC(Cc1ccccc1)C(=O)O</smiles>

Phenylalanine<smiles>NC(CCC(=O)NC(CS)C(=O)NCC(=O)O)C(=O)O</smiles>

e:<smiles>CSSCC(NC(=O)CCC(N)C(=O)O)C(=O)NCC(=O)O</smiles><smiles>NC(CCC(=O)NC(CS)C(=O)NCC(=O)O)C(=O)O</smiles>

Glutathione disulfide

Figure S2. Fluorescence emission spectra of the BSA-Au NC solution (a) before and after being treated with Cys (b), GSH (c), Met (d), GSSH (e) and phenylalanine (f). $\lambda_{\mathrm{ex}}=405 \mathrm{~nm}$. 


\section{TEM image of the BSA-Au NC solution treated with Cys.}

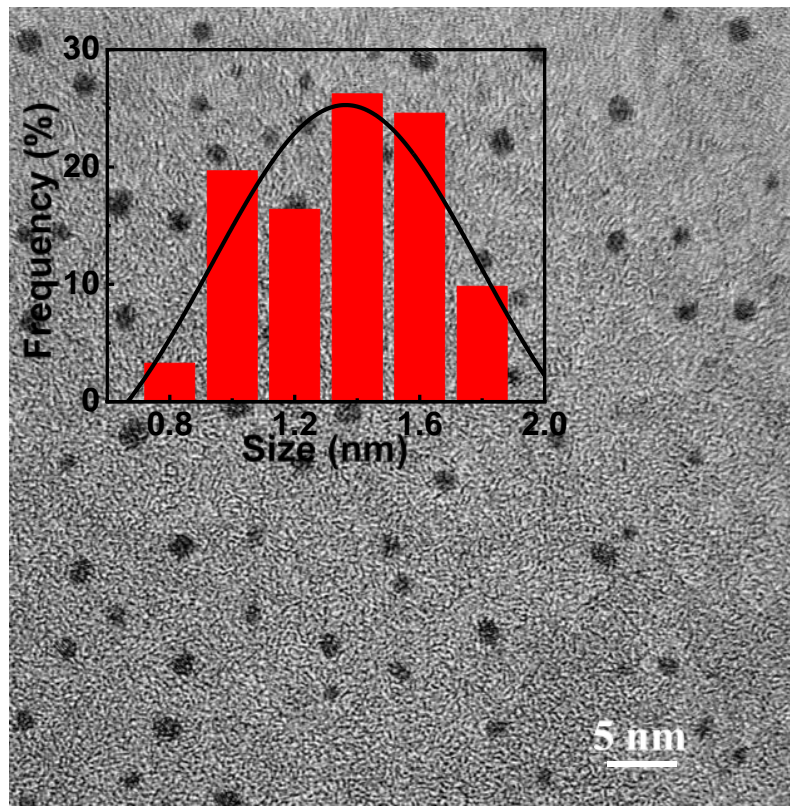

Figure S3. TEM image of BSA-AuNCs treated with Cys. (Insets) Size distribution histogram of BSA-AuNCs-Cys. 
4. Optical absorption spectra of the BSA-Au NC solution treated with Cys.

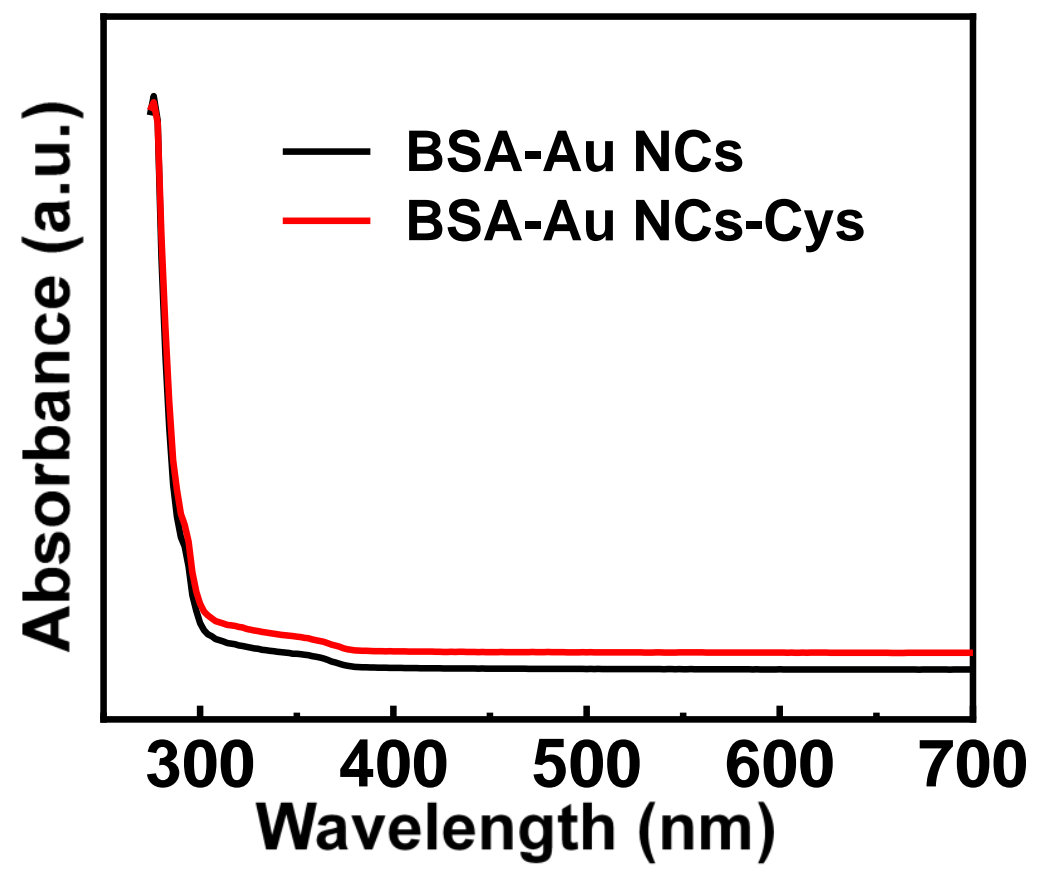

Figure S4. Optical absorption spectra of the BSA-Au NC solution before (black curve) and after (red curve) treatment with Cys. 


\section{SEM image}

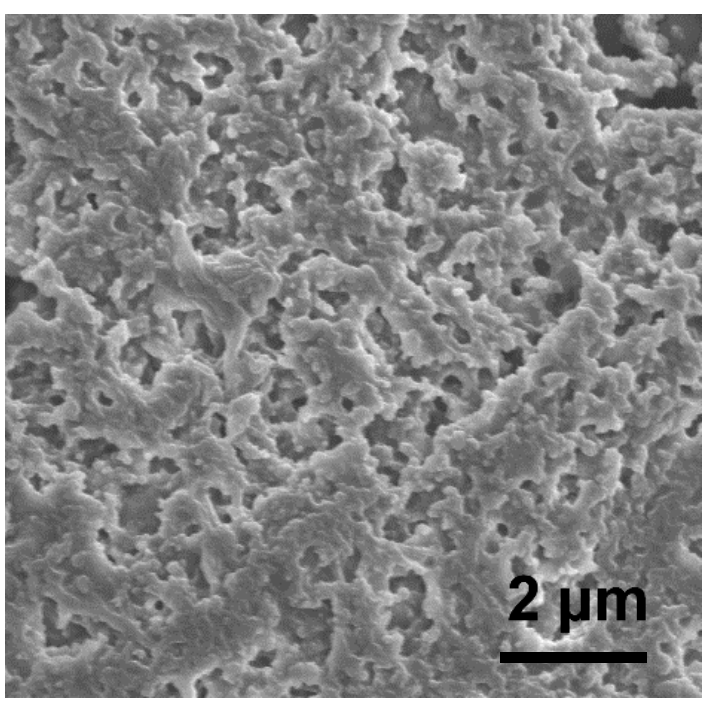

Figure S5. SEM image of PEI film-modified on planar glass. 
6. Optical absorption spectra of the BSA-Au NC film

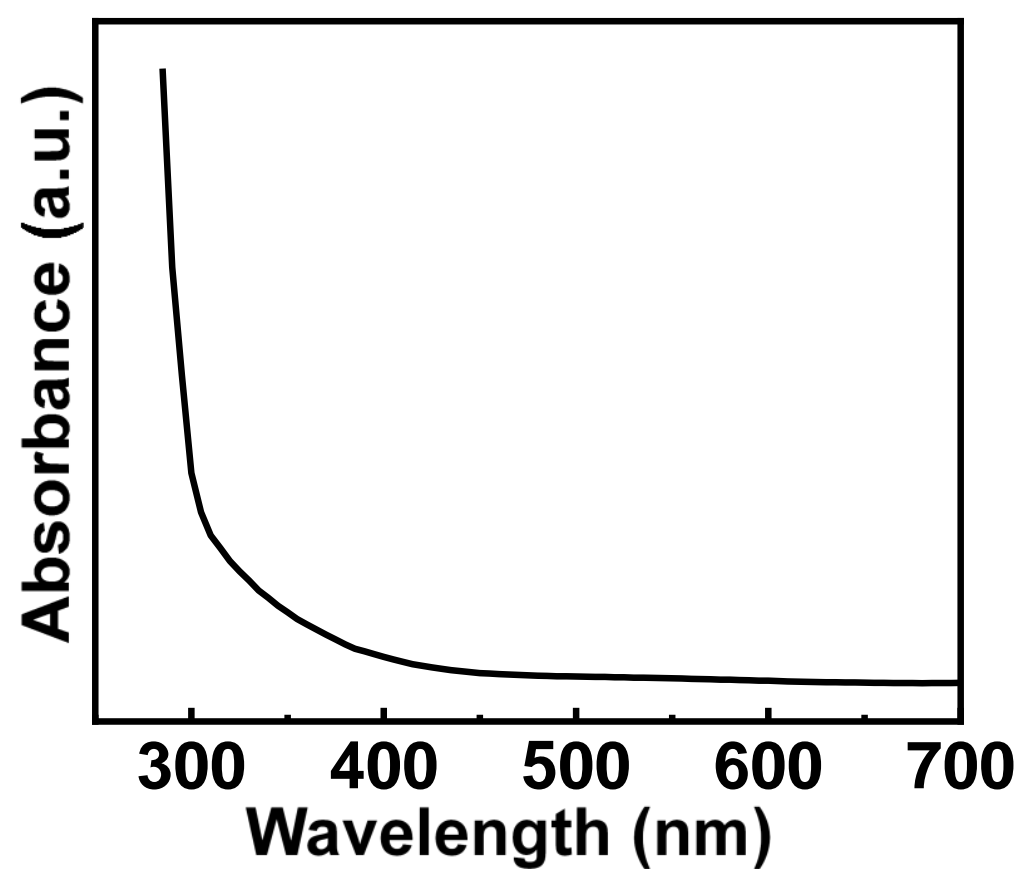

Figure S6. Optical absorption spectra of the BSA-AuNC film decorated on planar glass. 
7. Zeta potential of the BSA-Au NC film.

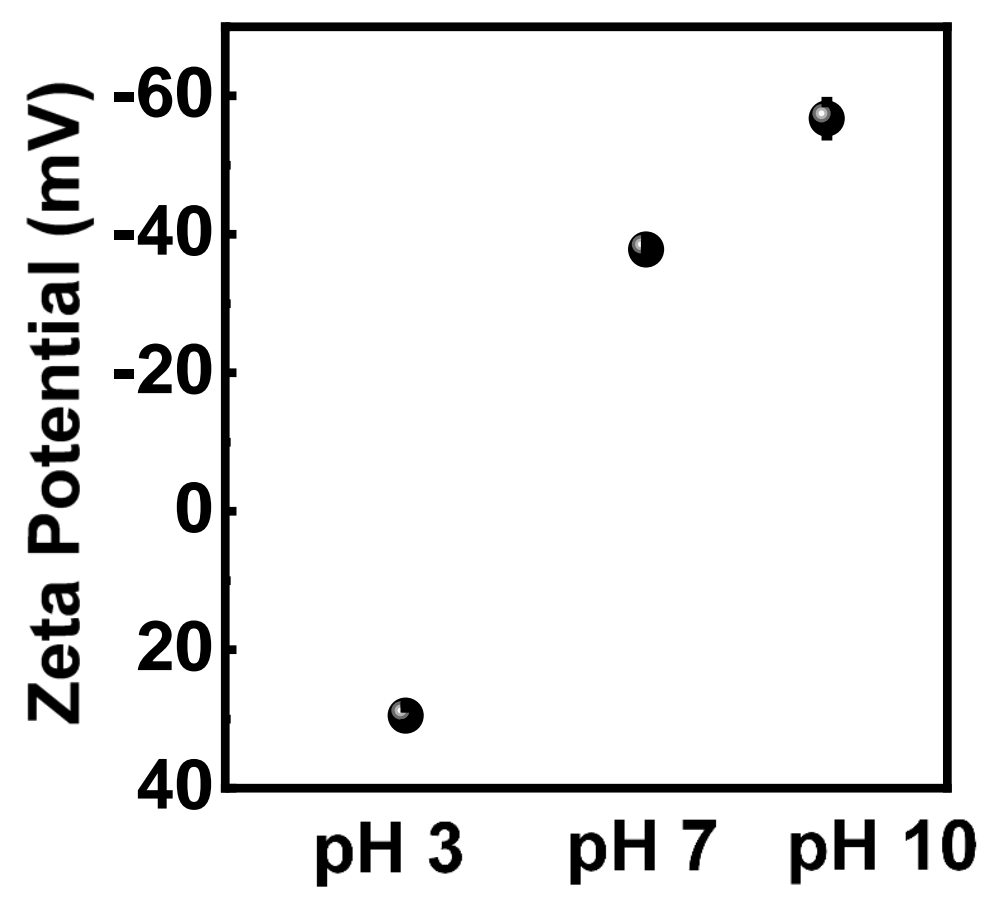

Figure S7. Zeta potential of the BSA-Au NC film prepared on the PEI-coated planar glass at different $\mathrm{pH}$. 


\section{SEM image.}

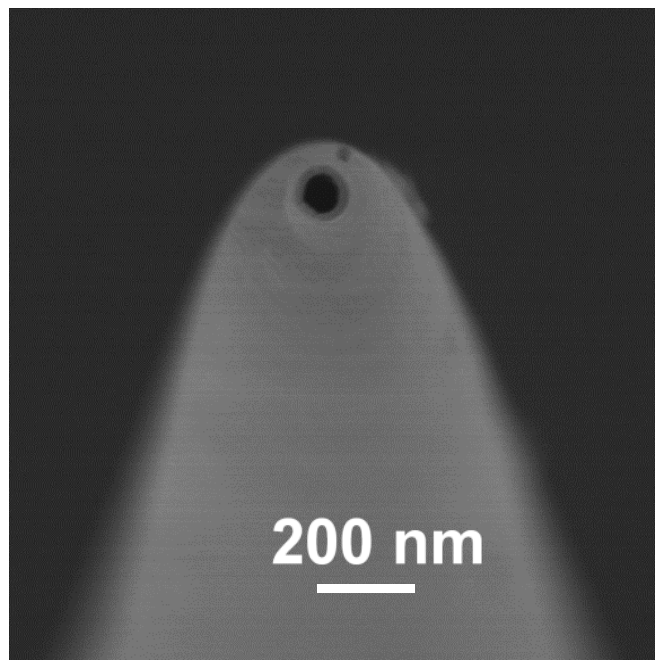

Figure S8. SEM image of the bare nanopore tip. 
9. $I-V$ curves.

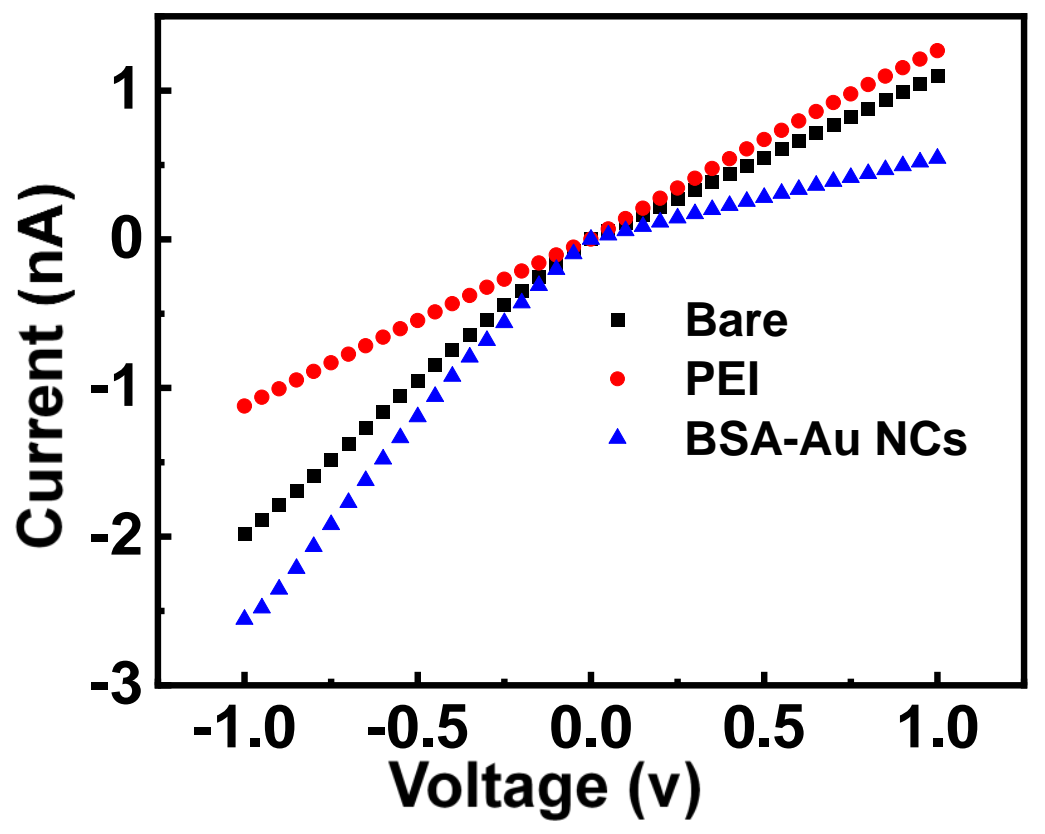

Figure S9. The $I-V$ curves of bare (black curve), PEI-coated (red curve) and the BSA-AuNC film-modified (blue curve) nanopore $(\sim 70 \mathrm{~nm})$ recorded in PBS (0.03 M, pH=7.0). 


\section{TEM images of glass nanopore}
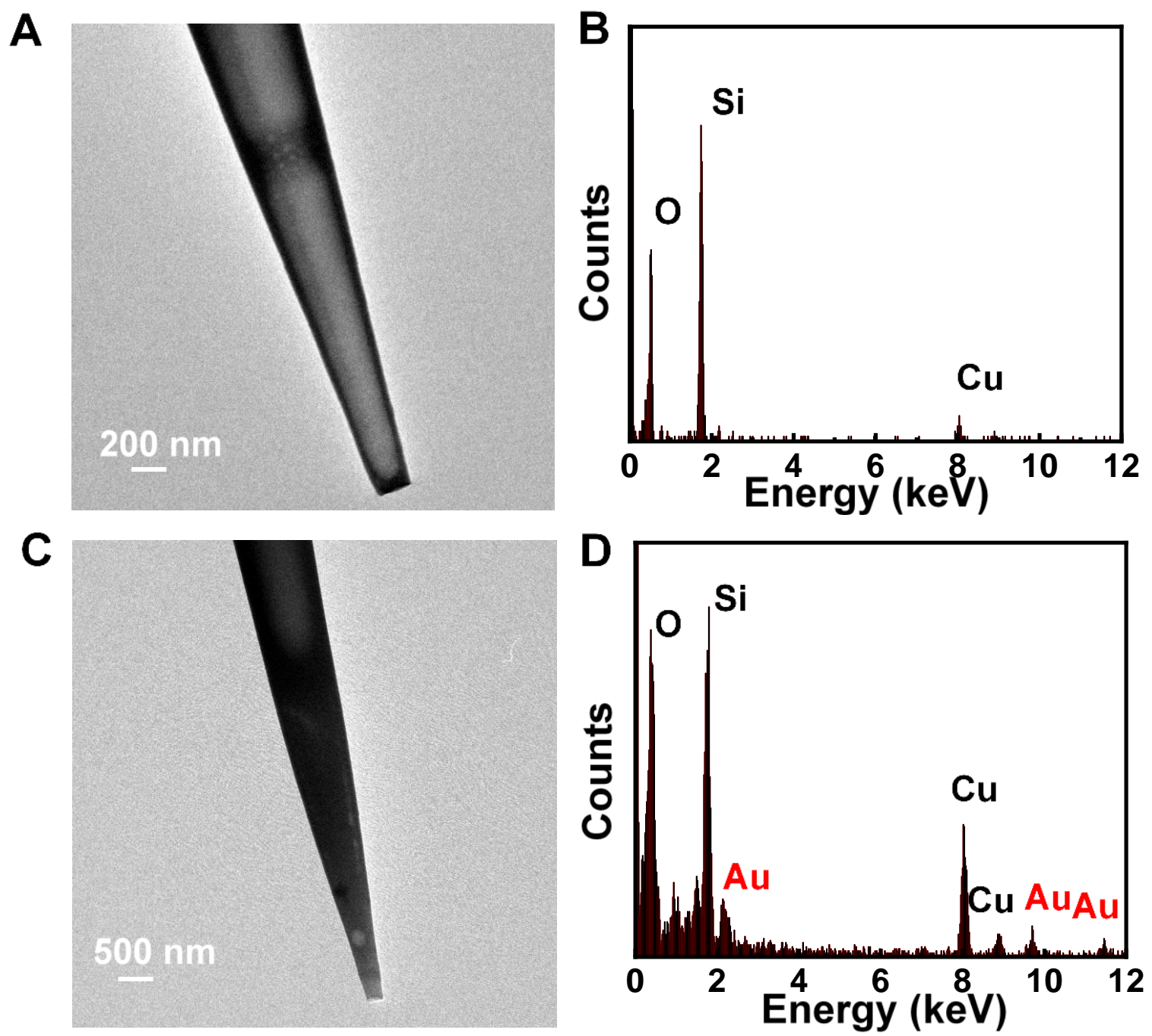

Figure S10. (A, C) TEM image and (B, D) EDX spectrum of $(\mathrm{A}, \mathrm{B})$ an unmodified glass nanopore tip and (C, D) a BSA-Au NC film-modified glass nanopore tip under low magnification. The glass nanopores with larger orifice diameter $(\sim 230 \mathrm{~nm})$ were employed. 


\section{Recognition of GSH}

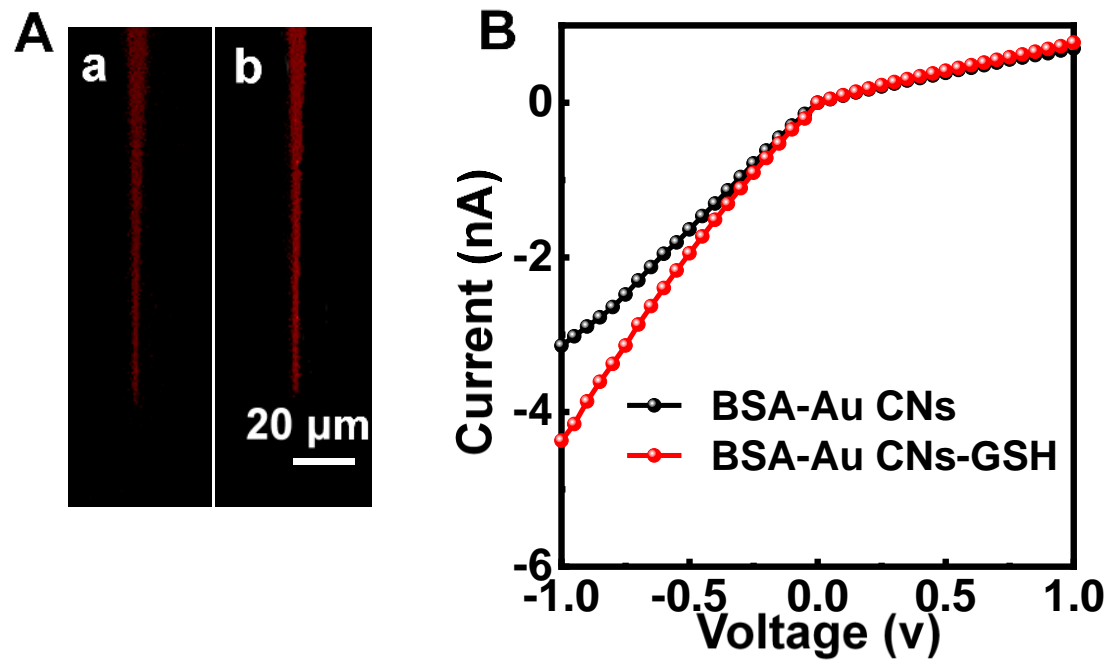

Figure S11. (A) The fluorescence images of the BSA-Au NC film-decorated glass nanopore before (a) and after (b) treatment with 1 pM GSH. (B) $I-V$ curves of the BSA-Au NC film-modified glass nanopore ( $\sim 80 \mathrm{~nm})$ measured in PBS (0.03 M, pH 7.0) before (black curve) and after (red curve) treatment with $1 \mathrm{pM}$ GSH. 


\section{Table S1 Comparison of the performance of different methods}

for the determination of cysteine

\begin{tabular}{|c|c|c|c|}
\hline Method & $\begin{array}{c}\text { Detection range } \\
(\boldsymbol{\mu} \mathbf{M})\end{array}$ & $\begin{array}{c}\text { Limit of detection } \\
(\mathbf{n M})\end{array}$ & Refs \\
\hline Colorimetric assay & $0.1-20$ & 100 & 1 \\
\hline Fluorometric assay & $1-40$ & 146 & 2 \\
\hline $\begin{array}{l}\text { Differential pulse } \\
\text { voltammetry }\end{array}$ & $0.25-100$ & 0.14 & 3 \\
\hline Nanopore analysis & $10^{-9}-10^{-6}$ & $10^{-6}$ & This work \\
\hline
\end{tabular}

\section{References}

(1) Du, J.; Wang, J.; Huang, W.; Deng, Y.; He, Y. Visible light-activatable oxidase mimic of 9-mesityl-10-methylacridinium ion for colorimetric detection of biothiols and logic operations. Anal. Chem., 2018, 90, 9959-9965.

(2) Gao, Q.; Zhang, W.; Song, B; Zhang, R.; Guo, W.; Yuan, J. Development of a novel lysosome-targeted ruthenium(II) complex for phosphorescence/timegated luminescence assay of biothiols. Anal. Chem., 2017, 89, 4517-4524.

(3) Balamurugan, T. S. T.; Huang, C. H.; Chang, P. C.; Huang, S. T.; Electrochemical Molecular Switch for the Selective Profiling of Cysteine in Live Cells and Whole Blood and for the Quantification of Aminoacylase-1. Anal. Chem., 2018, 90, 12631-12638. 